\title{
Tolerancia al Ejercicio en Perros con Hipertensión Pulmonar Tratados con Sildenafil
}

\author{
Exercise Tolerance in Dogs with Pulmonary Hypertension Treated \\ WITH SILDENAFIL \\ Pedro Vargas-Pinto ${ }^{1,4}$, Adriana Pedraza-Toscano ${ }^{2}$, Estefanía Betancourt ${ }^{1}$, \\ Christian González ${ }^{2}$, Piero Vargas-Pinto ${ }^{3}$
}

\section{Resumen}

Este estudio buscó establecer el efecto del sildenafil sobre la distancia recorrida en la prueba de marcha de seis minutos (6MWT) en caninos sanos y con hipertensión pulmonar (HP) sobre la frecuencia cardiaca (FC) y respiratoria (FR) en una zona de gran altitud (2650 m sobre el nivel del mar). Un grupo de nueve perros con diagnóstico de HP y otro de 10 perros clínicamente sanos hicieron la caminata en una pista plana de 30 metros, sin control de temperatura y humedad. Al final de la prueba se les administró 1 $\mathrm{mg} / \mathrm{kg}$ de sildenafil oral. Una hora después, se repitió la prueba. Antes y después de cada prueba se midió la FC y FR mediante auscultación. Los perros con HP caminaron $470 \pm$ 122.6 y $407.8 \pm 137.4 \mathrm{~m}$ antes y después de sildenafil, respectivamente $(\mathrm{p}=0.027)$. Asimismo, la FR fue más alta después de la prueba que antes de empezar $(\mathrm{p}<0.05)$. El grupo de caninos sanos caminó $560 \pm 55$ y $613 \pm 36$ m antes y después del sildenafil, respectivamente $(p=0.03)$ y redujo la FR antes y después de la segunda prueba donde recibieron sildenafil. Los perros con HP redujeron su tolerancia al ejercicio de manera drástica y presentaron aumentos de FR, mientras que los caninos sanos aumentaron la distancia recorrida con, posiblemente, menos requerimiento de oxígeno. Estos resultados ponen en duda la utilidad del sildenafil para la reducción de signos clínicos en caninos con hipertensión pulmonar en zonas de gran altura y sugieren su uso para mejorar la tolerancia aguda al ejercicio en caninos sanos en zonas de altura.

Palabras clave: prueba de marcha de seis minutos, sildenafil, gran altitud, hipertensión pulmonar

${ }^{1}$ Facultad de Ciencias Agropecuarias, Universidad de La Salle, Bogotá, Colombia

${ }^{2}$ Facultad de Medicina Veterinaria y Zootecnia, Universidad Antonio Nariño, Bogotá, Colombia

${ }^{3}$ Facultad de Medicina Veterinaria y de Zootecnia, Universidad Nacional de Colombia, Bogotá, Colombia

${ }^{4}$ E-mail: pavargas@unisalle.edu.co

Recibido: 23 de enero de 2016

Aceptado para publicación: 29 de abril de 2016 
This study aims to establish the effect of sildenafil on the distance covered during the six-minute-walk-test (6MWT) in dogs with pulmonary hypertension $(\mathrm{PH})$ and in healthy ones on heart rate (HR) and respiratory rate (RR) in a high altitude geographic zone (2650 $\mathrm{m}$ above sea level). A group of nine dogs diagnosed with PH and other of 10 healthy dogs walked in a flat track of 30 meters without control of temperature and humidity. After the test, dogs received $1 \mathrm{mg} / \mathrm{kg}$ of oral sildenafil. One hour later the test was repeated. HR and RR were measured before and after each test by auscultation. Dogs with $\mathrm{PH}$ walked $470 \pm 122.6$ and $407.8 \pm 137.4 \mathrm{~m}$ before and after sildenafil respectively $(\mathrm{p}=0.027)$ and the $\mathrm{RR}$ was higher after the walk than before the test $(\mathrm{p}<0.05)$. The group of healthy dogs walked $560 \pm 55$ and $613 \pm 363 \mathrm{~m}$ before and after sildenafil respectively $(p=0.03)$ and reduced the RR before and after the second test where received sildenafil. The dogs with PH drastically reduced the tolerance to exercise and showed increased $\mathrm{RR}$, while the healthy dogs increased the walked distance possibly due to a reduction in oxygen requirements. These results challenge the usefulness of sildenafil in the reduction of clinical signs in dogs with PH living in high altitude areas and suggest its use to improve tolerance to acute exercise in healthy dogs.

Key words: six-minute walk test, sildenafil, high altitude, pulmonary hypertension

\section{INTRODUCCIÓN}

La vida en áreas geográficas de altura representa un reto fisiológico grande para el sistema respiratorio y cardiovascular, pudiendo generarse alteraciones tales como reducción en el consumo de oxígeno con menor disponibilidad mitocondrial y mayor fatiga (West, 2004). En zonas de altitud, la población animal tiene un mayor riesgo de presentar hipertensión pulmonar dada la baja presión de oxígeno ambiental, ya que esta condición puede agravar el proceso de aumento de presión de la arteria pulmonar (Luks, 2009).

La hipertensión pulmonar (HP) es una condición patológica considerada emergente (Machado y Gladwin, 2010). Se caracteriza por el incremento progresivo de la resistencia y presión vascular pulmonar, que puede llevar a una falla ventricular derecha y disminución de la expectativa de vida (Simonneau et al., 2004). En perros, la causa más común es la complicación originada por la insuficiencia cardiaca izquierda, principalmente debido a insuficiencia valvular mitral de tipo mixomatoso (Chiavegato et al., 2009; Borgarelli et al., 2015). En medicina veterinaria, esta condición ha sido reportada con mayor frecuencia en perros, posiblemente debido a una mayor facilidad de identificar incrementos en la presión ventricular derecha mediante la interrogación por Doppler de la regurgitación de la tricúspide (Schober y Baade, 2006).

Se han identificado compuestos con diferentes mecanismos de acción para generar aumentos en la dilatación vascular pulmonar. Entre estos se encuentra el sildenafil, un inhibidor de la fosfodiesterasa 5 (Fox et al., 2003). Este medicamento genera reducciones en la poscarga ventricular derecha y ha sido utilizado para el tratamiento de la HP (Barnett y Machado 2006) y el edema pulmonar no cardiogénico (Kellihan et al., 2015). Adicionalmente, su uso se ha extendido a mejorar la tolerancia al ejercicio en mediana y gran altura (Rubin y Naeije 2004; Hsu et al., 2006) y para el tratamiento de la disfunción eréctil en pacientes humanos (Fox et al., 2003). 
Existen diversos estudios sobre el uso del sildenafil en pacientes humanos con hipertensión pulmonar donde se hace referencia al incremento en distancias recorridas bajo la prueba de marcha de 6 minutos (six-minute walk test - 6MWT) (Collard et al., 2007; Rao et al., 2011). En caninos con HP no ha sido demostrado un efecto positivo en la reducción de la presión arterial aunque se mejora el cuadro clínico (Kellum y Stepien, 2007) y se aumenta la actividad física medida por acelerómetro (Brown et al., 2010).

El presente trabajo discute los hallazgos encontrados en un grupo de perros diagnosticados con HP en comparación con un grupo de perros sanos, habitantes de zonas de altura, ante la administración de sildenafil.

\section{Materiales y MéTOdos}

El estudio se realizó en la sede norte de la Universidad de La Salle, situada a $2650 \mathrm{msnm}$. Se trabajó con un grupo de nueve perros diagnosticados con HP mediante interrogación de la velocidad de regurgitación de la tricúspide y que se encontraban medicados con sildenafil. Estos animales presentaban HP debido a insuficiencia cardiaca izquierda y estaban categorizados entre $\mathrm{A}$ y $\mathrm{C}$ de acuerdo a la escala de clasificación reportada para pacientes caninos con insuficiencia cardiaca (Atkins et al., 2009), sin hacer distinción por severidad del cuadro clínico.

Los pacientes fueron reclutados para el estudio y este proceso tomó 14 meses (septiembre de 2014 a noviembre de 2015). Los perros tenían entre 7 y 12 años de edad. De los nueve perros, cinco eran French Poodle o cruce con French Poodle, dos Schnawzer y dos considerados como criollos. Asimismo, se trabajó durante dos meses con un grupo de 10 perros considerados clínicamente sanos, que estuvo compuesto por cuatro Pitbull, dos Labrador Dorado, dos criollos y dos Schnawzer, con edades entre 1 y 6 años, y de sexo macho o hembra no gestante.

El protocolo de trabajo para los perros con HP fue aprobado por el comité de bioética de la Universidad de La Salle el 29 de agosto de 2014 mediante Acta N. ${ }^{\circ} 101$. Los propietarios fueron informados sobre el uso de la prueba como método diagnóstico y pronóstico en insuficiencia cardiaca e hipertensión pulmonar y fue incluido dentro del plan diagnóstico de los pacientes. En el caso de los caninos sanos, el protocolo fue aprobado por la Facultad de Medicina Veterinaria de la Universidad Antonio Nariño, ubicada en Bogotá, Colombia. En forma similar, los propietarios fueron informados sobre la prueba y firmaron una carta de aceptación de participación en el estudio.

Los perros de ambos grupos pasaron por la prueba de marcha de 6 minutos (6MWT) en una pista de 30 metros al aire libre. Los perros con HP no recibieron sildenafil las 24 horas previas a la prueba. Los pacientes fueron llevados al sitio de caminata y se les permitió descansar por un mínimo de cinco minutos y luego se les midió la frecuencia cardiaca y respiratoria por auscultación. Los perros hicieron las caminatas por seis minutos guiados por sus propietario y sujetos con collar (Swimmer y Rozanski, 2011). Los animales caminaron a su propio ritmo y velocidad, y se les permitió detenerse a descansar si era necesario. Al final de la prueba, se volvió a medir la frecuencia respiratoria y cardiaca, y se les administró una dosis de $1 \mathrm{mg} / \mathrm{kg}$ de sildenafil genérico vía oral. Los pacientes descansaron por una hora y luego se repitió el proceso de mediciones y la prueba de marcha. Las distancias recorridas fueron medidas con odómetro.

Se compararon los resultados de frecuencia cardiaca, frecuencia respiratoria y distancia recorrida antes y después de la administración de sildenafil. Se hicieron pruebas de normalidad con los datos y se compararon mediante prueba $\mathrm{T}$ y de análisis de varianza para establecer diferencias estadísticas. El test de Bonferroni se empleó para 
establecer diferencias entre los puntos de evaluación. Valores de $\mathrm{p}$ inferiores a 0.05 fueron considerados estadísticamente significativos.

\section{Resultados}

Los pacientes con HP (grupo 1) caminaron una distancia menor después de recibir la dosis de sildenafil $(407.8 \pm 137.4 \mathrm{~m})$ comparado con la distancia recorrida antes de recibir el medicamento $(470 \pm 122.6$; $\mathrm{p}=0.027$; Figura 1).

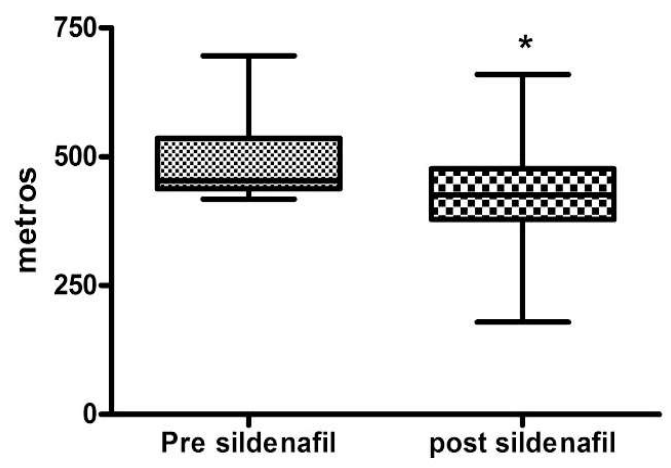

Figura 1. Distancias recorridas por perros con hipertensión pulmonar, habitantes en zona de altura (2650 $\mathrm{msnm})$, antes y después de recibir sildenafil $(\mathrm{p}<0.05)$

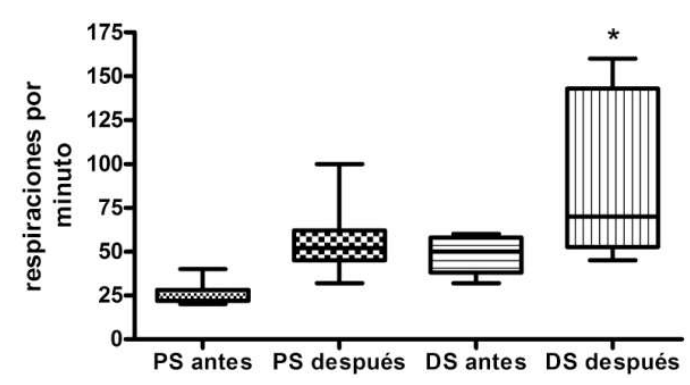

Figura 2. Frecuencia respiratoria con y sin sildenafil antes y después de la prueba de marcha de 6 minutos en pacientes con hipertensión pulmonar. La gráfica muestra una diferencia estadística $\left(^{*}\right)$ en frecuencia respiratoria en los perros con hipertensión pulmonar antes de la prueba de marcha sin sildenafil y después de la prueba de marcha con sildenafil. $P S$ : antes de sildenafil; $D S$ : después de sildenafil
La frecuencia respiratoria se incrementó de manera no significativa en los pacientes después de recibir la dosis de sildenafil. La frecuencia respiratoria antes de la prueba de marcha pasó de $30.57 \pm 11.47 \mathrm{rpm}$ sin sildenafil a $52.29 \pm 13.19 \mathrm{rpm}$ con sildenafil. Al terminar la prueba, la frecuencia respiratoria fue $60.43 \pm 18.4 \mathrm{rpm}$ sin sildenafil y 89.5 $\pm 41.4 \mathrm{rpm}$ con sildenafil. La frecuencia respiratoria no fue diferente entre el antes y después de la prueba sin sildenafil; sin embargo, al suministrar sildenafil se incrementó después de la prueba en comparación a antes de la misma ( $<<0.05$; Figura 2). No hubo cambios significativos en frecuencia cardiaca en ninguno de los puntos.

Los pacientes sanos (grupo 2) caminaron $560 \pm 55 \mathrm{~m}$ antes de sildenafil vs. $613 \pm$ $36 \mathrm{~m}$ después de sildenafil ( $\mathrm{p}=0.03$ ). La frecuencia respiratoria fue estadísticamente más baja una hora después del sildenafil (30.0 \pm 7) que antes de la primera prueba sin sildenafil $(27.0 \pm 7 ; \mathrm{p}<0.05)$. Asimismo, la frecuencia respiratoria después de la segunda prueba fue menor que aquella después de la primera prueba sin sildenafil ( $40.0 \pm 5$ vs. $46.0 \pm 15$; $\mathrm{p}<0.05$ ).

\section{Discusión}

Este estudio demuestra una reducción en la tolerancia al ejercicio en pacientes con HP clínica de diferente severidad en habitantes de zonas de altura, determinada mediante la prueba 6MWT, después de la administración de una dosis terapéutica de sildenafil. Asimismo, muestra un incremento en la frecuencia respiratoria al finalizar la prueba en los pacientes que tomaron sildenafil mientras que no hubo cambios de importancia cuando se realizó la prueba sin administrar el medicamento.

Si bien la prueba fue tolerada por todos los pacientes que participaron en el estudio, se puede considerar que los pacientes no solo caminaron distancias menores, sino que sus 
requerimientos de oxígeno pudieron haber aumentado. Esto podría deberse a un aumento en el retorno venoso hacia el ventrículo izquierdo debido a la reducción en la poscarga ventricular derecha y, como consecuencia de esto, un aumento en las presiones de llenado ventricular izquierdo y en las venas pulmonares.

En el caso de los animales sanos, se demostró un incremento significativo en las distancias recorridas en 6MWT después de sildenafil con lo que se puede considerar que hubo una reducción en los requerimientos de oxígeno, lo que podría deberse a un mejor flujo sanguíneo pulmonar con mejor intercambio gaseoso.

Estos resultados abren la incógnita sobre la utilidad o incluso el riesgo del uso de sildenafil en pacientes con hipertensión pulmonar en gran altura y plantea la posibilidad de que el sildenafil mejore la tolerancia al ejercicio y las respuestas fisiológicas asociadas a este en pacientes sanos en gran altura.

\section{Literatura Citada}

1. Atkins C, Bonagura J, EttingerS, Fox P, Gordon S, Haggstrom J, Hamlin R, et al. 2009. Guidelines for the diagnosis and treatment of canine chronic valvular heart disease. J Vet Intern Med 23: 11421150. doi: 10.1111/j.1939-1676.2009.0392.x

2. Barnett CF, Machado RF. 2006. Sildenafil in the treatment of pulmonary hypertension. Vasc Health Risk Manag 2: 411-422.

3. Borgarelli M, Abbott J, Braz-Ruivo L, Chiavegato D, Crosara S, Lamb K, Ljungvall I, et al. 2015. Prevalence and prognostic importance of pulmonary hypertension in dogs with myxomatous mitral valve disease. J Vet Intern Med 29: 569-574. doi: 10.1111/jvim. 12564
4. Brown AJ, Davison E, Sleeper MM. 2010. Clinical efficacy of sildenafil in treatment of pulmonary arterial hypertension in dogs. J Vet Intern Med 24: 850-854. doi: 10.1111/j.19391676.2010.0517.x

5. Chiavegato D, Borgarelli $M$, D'Agnolo G, Santilli RA. 2009. Pulmonary hypertension in dogs with mitral regurgitation attributable to myxomatous valve disease. Vet Radiol Ultrasound 50: 253-258. doi: 10.1111/ j.1740-8261.2009.01529.x

6. Collard HR, Anstrom KJ, Schwarz MI, Zisman DA. 2007. Sildenafil improves walk distance in idiopathic pulmonary fibrosis. Chest 131: 897-899. doi: 10.1378/chest.06-2101

7. Fox KM, Thadani U, Ma PT, Nash SD, Keating Z, Czorniak MA, Gillies $H$, et al. 2003. Sildenafil citrate does not reduce exercise tolerance in men with erectile dysfunction and chronic stable angina. Eur Heart J 24: 2206-2212. doi: 10.1016/j.ehj.2003.09.021

8. Hsu AR, Barnholt KE, Grundmann NK, Lin JH, McCallum SW, Friedlander AL. 2006. Sildenafil improves cardiac output and exercise performance during acute hypoxia, but not normoxia. J Appl Physiol 100: 20312040. doi: 10.1152/japplphysiol. 00806.2005

9. Kellihan HB, Waller KR, Pinkos A, Steinberg H, Bates ML. 2015. Acute resolution of pulmonary alveolar infiltrates in 10 dogs with pulmonary hypertension treated with sildenafil citrate: 2005-2014. J Vet Cardiol 17: 182191. doi: 10.1016/j.jvc.2015.04.002

10. Kellum HB, Stepien RL. 2007. Sildenafil citrate therapy in 22 dogs with pulmonary hypertension. J Vet Intern Med 21: 1258-1264.

11. Luks AM. 2009. Can patients with pulmonary hypertension travel to high altitude? High Alt Med Biol 10: 215-219. doi: 10.1089/ham.2009.10306 
12. Machado RF, Gladwin MT. 2010. Pulmonary hypertension in hemolytic disorders: pulmonary vascular disease: the global perspective. Chest 137(6 Suppl): 30S-38S. doi: 10.1378/chest.093057

13. Rao RS, Singh S, Sharma BB, Agarwal VV, Singh V. 2011. Sildenafil improves six-minute walk distance in chronic obstructive pulmonary disease: a randomised, double-blind, placebocontrolled trial. Indian J Chest Dis Allied Sci 53: 81-85.

14. Rubin LJ, Naeije R. 2004. Sildenafil for enhanced performance at high altitude? Ann Intern Med 141: 233-235. doi: 10.7326/0003-4819-141-3-20040 8030-00016
15. Schober KE, Baade H. 2006. Doppler echocardiographic prediction of pulmonary hypertension in West Highland white terriers with chronic pulmonary disease. J Vet Intern Med 20: 912-920. doi: 10.1111/ j.1939-1676.2006.tb01805.x

16. Simonneau G, Galie N, Rubin LJ, Langleben D, Seeger W, Domenighetti G, Gibbs S, et al. 2004. Clinical classification of pulmonary hypertension. J Am Coll Cardiol 43: 5S-S12. doi:10.1016/j.jacc.2004.02.037

17. Swimmer RA, Rozanski EA. 2011. Evaluation of the 6-minute walk test in pet dogs. J Vet Intern Med 25: 405-406. doi: 10.1111/j.1939-1676.2011.0689.x

18. West JB. 2004. The physiologic basis of high-altitude diseases. Ann Intern Med 141: 789-800. 